\title{
Cerebral aneurysms and accelerated atherosclerosis in Russell-Silver syndrome: a new subtype? Case report
}

\author{
Heinke Pülhorn, MD, FRCS, ${ }^{1}$ Jonathan Pesic-Smith, MBBS, MRCS, ${ }^{2}$ Peter Cowley, MD, FRCR, ${ }^{3}$ \\ and Mary Murphy, MD, FRCS ${ }^{1}$ \\ ${ }^{1}$ Victor Horsley Department of Neurosurgery, The National Hospital for Neurology and Neurosurgery; ${ }^{2}$ Whittington Hospital \\ NHS Trust; and ${ }^{3}$ Lysholm Department of Neuroradiology, The National Hospital for Neurology and Neurosurgery, London, \\ United Kingdom
}

\begin{abstract}
The authors describe the case of a 32-year-old woman known to have Russell-Silver syndrome who presented with repeated aneurysmal subarachnoid hemorrhage. Multiple intracranial aneurysms and advanced peripheral vascular disease were demonstrated. The authors postulate a link between these vascular features and the patient's genetic condition.
\end{abstract}

http://thejns.org/doi/abs/10.3171/2014.11.JNS1410

KEY WORDS Russell-Silver syndrome; aneurysm; atherosclerosis; subarachnoid hemorrhage; vascular disorders

$\mathrm{R}$ USSELL-SILVER syndrome (RSS) is a rare congenital imprinting disorder that leads to intrauterine growth restriction, low birth weight, and, in adulthood, significantly reduced body height. ${ }^{7,11}$ It was first described by Silver et al. ${ }^{9}$ in 1953 and, independently, 1 year later by Russell. ${ }^{8}$ Signs and symptoms of RSS include birth weight $<2.8 \mathrm{~kg}$, feeding problems, hypoglycemia, triangular face with a small jaw, pointed chin, downturned corners of the mouth, blue tinge to the sclera, asymmetrical body growth, late-closing fontanels, clinodactyly, gastroesophageal reflux disease, lack of subcutaneous fat, constipation, increased sweating, and decreased muscle bulk. It can also be associated with delayed development and impaired cognitive abilities. ${ }^{10}$ There have recently been reports of complex cardiac abnormalities. ${ }^{6,10}$ However, peripheral vascular abnormalities have never been described as a defining feature of RSS.

The exact cause of RSS is unknown. Its incidence is approximately $1 / 50,000$ to $1 / 100,000$. Most cases of RSS arise sporadically. However, $10 \%$ of patients have uniparental maternal disomy of chromosome $7,3,4$ and at least $44 \%$ of patients with RSS show hypomethylation in the chromosome $11 \mathrm{p} 15$ imprinting control region $1 .{ }^{5}$ Furthermore, a small number of reports of autosomal dominant transmission including ring 2 chromosome, balanced translocation of 17q25, and duplication of band 7p11.2-p13 have been published. ${ }^{3}$ Genetic testing for the syndrome is in development, ${ }^{4}$ but the pathophysiological mechanisms resulting in the RSS phenotype remain unknown.

We report on an unusual case of widespread accelerated atherosclerosis and multiple cerebral aneurysms in a patient with RSS. Written informed consent was obtained from the patient prior to publication of this case report.

\section{Case Report}

Presentation and History

A 32-year-old woman presented to the emergency department with a 2-day history of sudden-onset headache, confusion, and vomiting. She was known to have RSS and, when she was 25 years of age, had suffered a subarachnoid hemorrhage (SAH), due to a left middle cerebral artery (MCA) aneurysm, which had subsequently been clipped.

The patient's medical history indicated that she had received growth hormone therapy as a child. She was a cigarette smoker of 8 pack-years.

In terms of family history, the patient is the third child of consanguineous parents. Her parents are second cousins, and her mother's parents were first cousins. Her two older male siblings are genetically normal, but a 29 -year-

ABBREVIATIONS LDL = low-density lipoprotein; MCA = middle cerebral artery; $\mathrm{RSS}=$ Russell-Silver syndrome; SAH = subarachnoid hemorrhage SUBMITTED January 2, 2014. ACCEPTED November 13, 2014

INCLUDE WHEN CITING Published online January 2, 2015; DOI: 10.3171/2014.11.JNS1410.

DISCLOSURE The authors report no conflict of interest concerning the materials or methods used in this study or the findings specified in this paper. 
old younger brother also has RSS. In his case, however, arterial imaging has not shown any vascular abnormality.

\section{Examination}

The patient's Glasgow Coma Scale score was 14/15 (eye opening spontaneously, verbal response confused, motor response obeying commands). No focal neurological abnormality was detected.

Head CT scanning confirmed SAH. The SAH was shown to be Grade II on the World Federation of Neurosurgical Societies scale and Grade 1 on the Fisher scale. CT angiography and subsequent digital subtraction angiography of the circle of Willis showed a large complex right proximal MCA aneurysm and a smaller right distal MCA aneurysm (Fig. 1). Furthermore, an aneurysm was observed at the basilar tip and another on the superior cerebellar artery. Extracranially, whole-body CT angiography revealed heavy calcification with 50\% narrowing of both common carotid arteries just proximal to the carotid artery bulbs. Distally, the common femoral, external iliac, and internal iliac arteries were patent bilaterally but exhibited reduced caliber, and an aortoiliac occlusion was seen to extend from just below the origin of the internal mesenteric artery to the external iliac artery origins bilaterally.

The patient underwent successful clipping of the com-

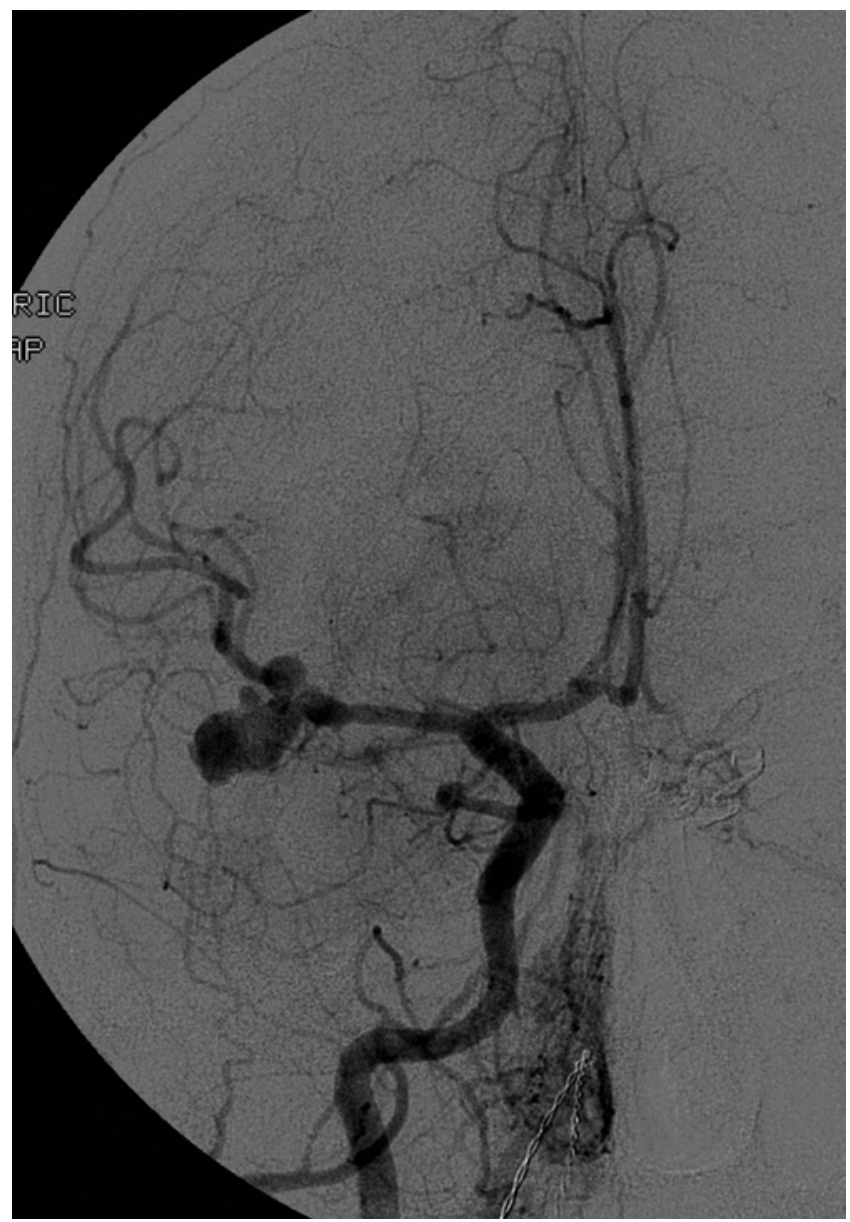

FIG. 1. Right MCA angiogram acquired after aneurysm reclipping. plex right proximal MCA aneurysm, from which she made a good recovery. Four months later, coiling of the basilar tip and superior cerebellar artery aneurysms was successfully carried out. The smaller, more distal MCA aneurysm remains, for the time being, untreated.

The patient has been under surveillance follow-up for her treated and untreated aneurysms for 5 years; surveillance involves a combination of MRI, MR angiography, and iv Dyna-CT scanning. She is clinically well, the treated aneurysms are stable, and the untreated distal MCA aneurysm remains unchanged in size and configuration.

\section{Discussion}

This report describes the presentation and successful management of a young patient with RSS complicated by multiple cerebral aneurysms and widespread accelerated vascular disease.

Cerebral aneurysms are rare in young patients. They typically produce symptoms in people aged $40-60$ years with the peak incidence of SAH occurring in those aged 55-60 years. ${ }^{1}$ These lesions may present earlier in association with predisposing genetic conditions, such as connective tissue disorders, fibromuscular dysplasia, spontaneous dissections, and autosomal dominant polycystic kidney disorder. However, RSS is not known to have such a predisposition. As mentioned before, our patient's brother, who also has RSS, has no features of vascular disease.

Early atherosclerosis also develops from conditions associated with high cholesterol such as familial hypercholesterolemia. This condition is often associated with a mutation in the low-density lipoprotein (LDL) receptor protein, which is involved in the removal of LDL cholesterol from the circulation. This results in high levels of LDL, which in turn is deposited in arterial walls and leads to cardiovascular and peripheral vascular disease. Cerebral involvement most commonly takes the form of transient ischemic attacks and strokes rather than aneurysmal dilation. Cholesterol levels were normal in our patient and investigations into other conditions predisposing to vascular disease or aneurysms have been negative. On medical advice, the patient has stopped smoking.

RSS is known to occur sporadically and the epigenetic implications are only just being discovered. ${ }^{3-5}$ However, in some cases dominant inheritance may play a role. ${ }^{2}$ Whether the fact that our patient's parents are consanguineous was instrumental to the development of RSS remains open to speculation. Neither of the parents is phenotypically abnormal.

Our patient shows some of the typical features of RSS in her phenotype, with a small body height of $123 \mathrm{~cm}$, a triangular face, a pointed chin, down-turned corners of the mouth, and blue-tinged sclera. She does not have any asymmetrical body growth and has no cardiac abnormalities. Genetic testing for RSS was performed on her and her younger brother in childhood, but as this was done abroad the results are not available to us.

We cannot rule out the possibility that the described vascular aging and the development of multiple intracranial aneurysms is coincidental in this patient with RSS. However, despite wide-ranging investigations, we have 
failed to identify any conditions in our patient that would predispose her to the detected vascular changes.

Until now, cardiovascular involvement in RSS has been limited to various cardiac abnormalities. To our knowledge, this is the first documented case of associated vascular pathology; however, the possible physiological basis underlying this association remains a mystery.

If the intra- and extracranial vascular features found in our patient are due to the genetic abnormalities associated with RSS, they define a new subtype of the syndrome. This would mean that vascular imaging should be performed in newly diagnosed cases of RSS to confirm the presence or absence of this subtype. Also, a screening program should be initiated to detect and treat vascular abnormalities in these patients early. Lifestyle advice with regard to smoking, blood pressure control, and cholesterol control should be encouraged. Furthermore, the presence of multiple intracranial aneurysms would then in turn be an additional way of diagnosing RSS.

\section{Conclusions}

We postulate a subtype of RSS in which accelerated vascular aging predisposing to aneurysm development as well as peripheral vascular disease is present in addition to the known features of the disease. The implications for screening and treating these vascular abnormalities are far reaching.

\section{References}

1. Brisman JL: Neurosurgery for cerebral aneurysm. Medscape. (http://emedicine.medscape.com/article/252142-overview) [Accessed November 16, 2014]

2. Duncan PA, Hall JG, Shapiro LR, Vibert BK: Three-generation dominant transmission of the Silver-Russell syndrome. Am J Med Genet 35:245-250, 1990

3. Eggermann T: Russell-Silver syndrome. Am J Med Genet C Semin Med Genet 154C:355-364, 2010
4. Eggermann T, Spengler S, Gogiel M, Begemann M, Elbracht M: Epigenetic and genetic diagnosis of Silver-Russell syndrome. Expert Rev Mol Diagn 12:459-471, 2012

5. Eggerman T, Wollmann HA, Kuner R, Eggermann K, Enders $\mathrm{H}$, Kaiser P, et al: Molecular studies in 37 Silver-Russell syndrome patients: frequency and etiology of uniparental disomy. Hum Genet 100:425-429, 1997

6. Ghanim M, Rossignol S, Delobel B, Irving M, Miller O, Devisme L, et al: Possible association between complex congenital heart defects and 11p15 hypomethylation in three patients with severe Silver-Russell syndrome. Am J Med Genet A 161A:572-577, 2013

7. Lister Hill National Center for Biomedical Communications: Russell-Silver syndrome. Genetics Home Reference. (http:// ghr.nlm.nih.gov/condition=russellsilversyndrome) [Accessed November 16, 2014]

8. Russell A: A syndrome of intra-uterine dwarfism recognizable at birth with cranio-facial dysostosis, disproportionately short arms, and other anomalies (5 examples). Proc R Soc Med 47:1040-1044, 1954

9. Silver HK, Kiyasu W, George J, Deamer WC: Syndrome of congenital hemihypertrophy, shortness of stature, and elevated urinary gonadotropins. Pediatrics 12:368-376, 1953

10. Sinha S: Silver-Russell syndrome. Medscape. (http://emed icine.medscape.com/article/948786-overview) [Accessed November 16, 2014]

11. Stanhope R, Albanese A, Azcona C: Growth hormone treatment of Russell-Silver Syndrome. Horm Res 49 Suppl 2:37-40, 1998

\section{Author Contributions}

Conception and design: Pülhorn, Murphy. Drafting the article: Pülhorn, Pesic-Smith. Critically revising the article: Cowley, Murphy. Administrative/technical/material support: Cowley.

\section{Correspondence}

Heinke Pülhorn, Department of Neurosurgery, The National Hospital for Neurology and Neurosurgery, Queen Square, London WC1N3BG, United Kingdom. email: armadillo@ doctors.org.uk. 